\title{
INSTABILITÉ D'UNE NAPPE CYLINDRIQUE DE TOURBILLONS
}

\author{
N. BOCCARA, R. CONTE et G. SARMA \\ Service de Physique du Solide et de Résonance Magnétique \\ CEN-Saclay, BP no 2, 91190 Gif-sur-Yvette, France
}

\begin{abstract}
Résumé. - On examine la stabilité d'une nappe cylindrique de tourbillons relativement à de petites déformations, le fluide dans lequel est placée la nappe étant incompressible et parfait. L'étude numérique de ce problème plan conclut à l'instabilité : la nappe se scinde en un nombre d'amas tourbillonnaires inférieur à 7. Ce résultat expérimental est confirmé par l'étude des petits mouvements de la nappe au voisinage de la configuration non déformée : le spectre de fréquences de tourbillons disposés aux sommets d'un polygone régulier n'est réel que si les tourbillons sont en nombre inférieur à 7 .
\end{abstract}

Abstract. - We study the stability of a cylindrical vortex-sheet under small deformations, in an incompressible perfect fluid. Numerical study of this planar problem concludes in favour of instability : the sheet splits into a number of vortex clusters lesser than 7 . This experimental result is confirmed by the study of small motions of the sheet around the unperturbed configuration : the frequency spectrum of vortices set at the vertices of a regular polygon is real only if the number of vortices is lesser than 7 .

1. Introduction. - Considérons une nappe de tourbillons libres de révolution autour d'un axe et infinie dans la direction de cet axe, placée dans un fluide incompressible parfait au repos à l'infinj. Notons $\Gamma$ la circulation totale et $R$ le rayon d'une section droite de la nappe par un plan, que nous prenons pour plan $x y$.

Nous nous proposons d'étudier la stabilité de cette nappe relativement aux petites déformations respectant l'invariance par translation parallèle à $O z$.

Pour étudier numériquement l'évolution, nous discrétisons ce problème plan en représentant la nappe par un grand nombre $N$ de tourbillons ponctuels libres de même circulation $C=\Gamma / N$, disposés aux sommets d'un polygone régulier de $N$ côtés inscrit dans le cercle de rayon $R$. Chacun des tourbillons subit l'action du champ de vitesses de tous les autres et l'ensemble évolue donc suivant la loi :

$$
\begin{gathered}
\forall j=1,2, \ldots, N, \\
\frac{\mathrm{dr}_{j}}{\mathrm{~d} t}=\frac{\mathbf{C}}{2 \pi} A \sum_{l}^{\prime} \operatorname{grad}_{j} \log \left|\mathbf{r}_{j}-\mathbf{r}_{\boldsymbol{l}}\right|
\end{gathered}
$$

(' indique l'exclusion de $l=j$ de la sommation), soit encore, en notation complexe :

$$
\begin{gathered}
\forall j=1,2, \ldots, N, \\
\frac{\mathrm{d} z_{j}}{\mathrm{~d} t}=\frac{i C}{2 \pi} \sum_{l}^{\prime} \frac{1}{\bar{z}_{j}-\bar{z}_{l}} .
\end{gathered}
$$

Lorsqu'ils sont disposés comme il est indiqué cidessus, les centres des tourbillons sont animés d'un mouvement circulaire uniforme de pulsation :

$$
\omega=\left(1-\frac{1}{N}\right) \frac{\Gamma}{4 \pi R^{2}},
$$

et le polygone ne se déforme pas. Si l'on s'écarte légèrement de cette configuration, par exemple en imposant à chaque point un petit déplacement initial aléatoire, le système se met à évoluer.

A partir des relations :

$$
\sum_{j} \frac{\mathrm{d} z_{j}}{\mathrm{~d} t}=0 \quad \text { et } \quad \sum_{j} \bar{z}_{j} \frac{\mathrm{d} z_{j}}{\mathrm{~d} t}=(N-1) \frac{i \Gamma}{4 \pi}
$$

on déduit que les quantités suivantes sont des constantes du mouvement :

- le barycentre,

- le moment d'inertie par rapport à un point quelconque,

- le moment cinétique par rapport à un point quelconque, égal à

$$
(N-1) \frac{\Gamma}{4 \pi} .
$$

2. Etude expérimentale. - Nous avons simulé sur ordinateur l'évolution d'un grand nombre (100, puis 200) de ces tourbillons.

Le système différentiel (2) est résolu numériquement par la méthode du predictor-corrector de Hamming [4] avec ajustement du pas en temps selon la précision demandée. Les positions initiales (Fig. 1) sont obtenues par tirage au hasard suivant une loi uniforme dans un voisinage des sommets du polygone régulier ; ce voisinage est choisi de l'ordre de grandeur de la distance curviligne $a$ entre deux sommets voisins ; en effet, prendre des déplacements d'un ordre de grandeur inférieur n'aurait d'autre conséquence que d'augmenter le temps de calcul, sans changement ni qualitatif ni quantitatif des phénomènes. 


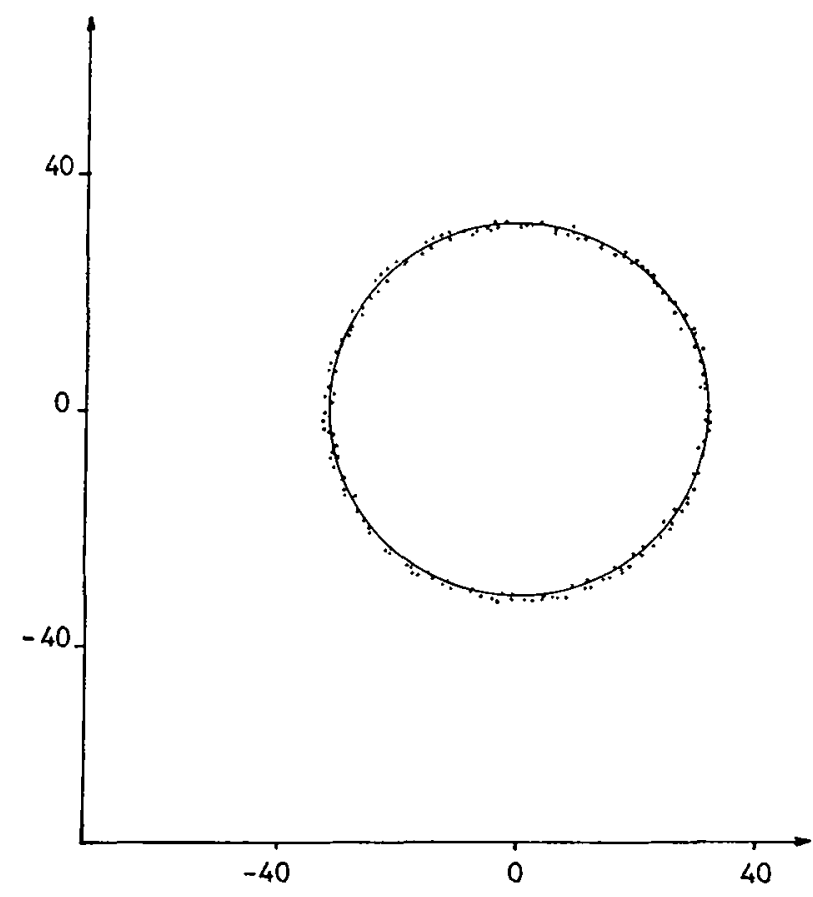

Fig. 1. - Configuration initiale 200 tourbillons, instant 0 .

Comme nous nous limitons à l'étude de la stabilité, du moins dans le présent article, nous ne mettons pas en œuvre des techniques de calcul élaborées [1-3] pour nous débarrasser d'un éventuel mouvement chaotique provenant du processus numérique de résolution; le critère d'exactitude des calculs est la constance des invariants.

L'expérience montre qu'après un régime transitoire dont la durée est de l'ordre de grandeur de $N \tau$, où $\tau$ est l'unité de temps $a^{2} / C$, il s'établit un régime permanent. Dans ce régime permanent, les tourbillons se sont groupés en un petit nombre de gros paquets (Fig. 2) animés en moyenne d'un mouvement circulaire uniforme ; autour de sa position moyenne, chaque paquet est animé d'oscillations dont les modes ont une période de l'ordre de grandeur de $N \tau$. De plus, le nombre final de paquets semble indépendant de $N$ et s'établit à une valeur de 5 ou de 6 , chaque paquet étant composé du même nombre de tourbillons. Sur la figure 3, où l'on a tracé l'histogramme du nombre de paires de tourbillons en fonction de l'angle sous lequel la paire est vue depuis le barycentre, la position du premier minimum, en $\pi / 6$, traduit le groupement en 6 paquets.

Les tourbillons constitutifs d'un paquet ont un mouvement chaotique mais cette particularité provient très certainement de la discrétisation du problème (voir remarque ci-dessus) et nous poursuivons actuellement les travaux en vue de tirer au clair la structure interne des paquets.

$\mathrm{Au}$ vu de ce phénomène de groupement, que la loi

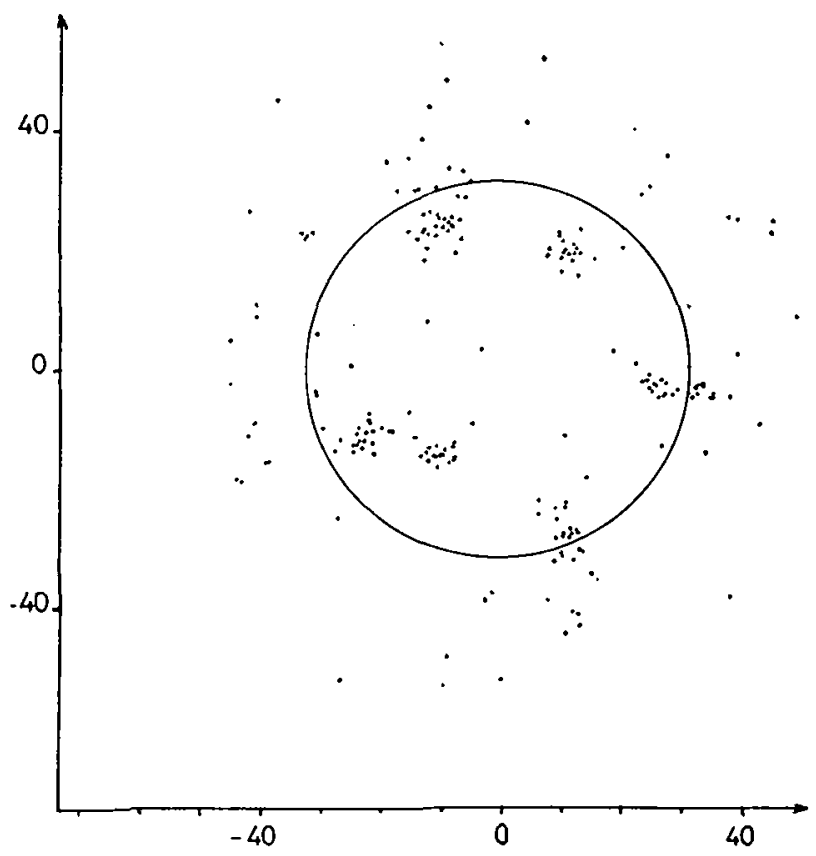

FIG. 2. -200 tourbillons, instant $1077 \tau$.

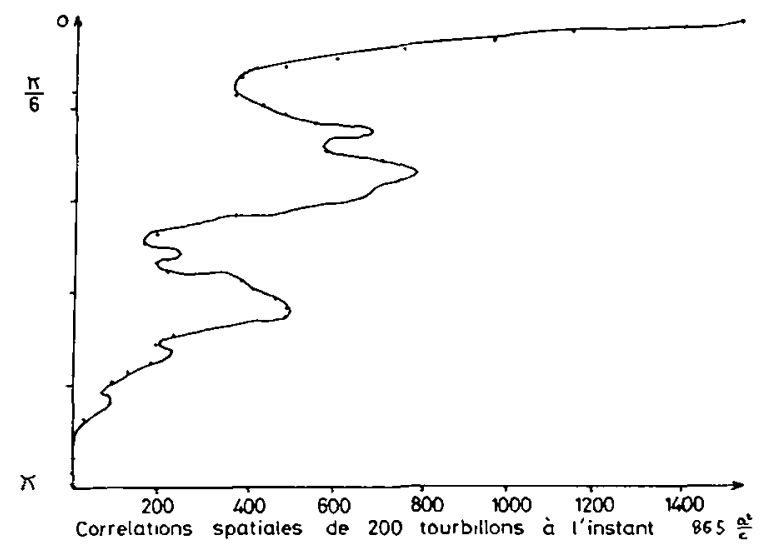

FIG. 3.

d'évolution et ses symétries ne laissaient nullement prévoir, on s'est demandé s'il n'existerait pas de constantes du mouvement cachées favorisant cette configuration. Le terme cachées signifie que le phénomène proviendrait non pas de considérations de symétrie mais de la forme analytique particulière des interactions.

3. Interprétation théorique. - Montrons tout d'abord que rien ne s'oppose à l'apparition de paquets. Supposons donc que $N$ tourbillons aient évolué vers $p$ paquets de chacun $n$ tourbillons et appelons $z_{k j}$ l'affixe du $j$-ième tourbillon du $k$-ième paquet et $Z_{k}$ l'affixe du barycentre du $k$-ième paquet. 
A partir des équations du mouvement

$$
\begin{gathered}
\forall k=1, \ldots, p, \quad \forall j=1, \ldots, n, \\
\frac{\mathrm{d} z_{k j}}{\mathrm{~d} t}=\frac{i C}{2 \pi} \sum_{m l}^{\prime} \frac{1}{\bar{z}_{k j}-\bar{z}_{m l}}
\end{gathered}
$$

cherchons l'évolution des barycentres des paquets:

$$
\begin{gathered}
\forall k=1, \ldots, p \\
\frac{\mathrm{d} Z_{k}}{\mathrm{~d} t}=\frac{i C}{2 \pi n} \sum_{j} \sum_{m l}^{\prime} \frac{1}{\bar{z}_{k j}-\bar{z}_{m l}} .
\end{gathered}
$$

Si l'on introduit les déplacements $\zeta_{k j}=z_{k j}-Z_{k}$ par rapport aux barycentres et qu'on développe en $\zeta$, les sommations dans (6) annulent les termes du premier ordre en $\zeta$; c'est donc au deuxième ordre en ces termes que l'on obtient :

$$
\begin{gathered}
\forall k=1, \ldots, p, \\
\frac{\mathrm{d} Z_{k}}{\mathrm{~d} t}=\frac{i n C}{2 \pi} \sum_{m}^{\prime} \frac{1}{\overline{\bar{Z}}_{k}-\bar{Z}_{m}}
\end{gathered}
$$

équation d'évolution de $p$ tourbillons de circulation $n C$, d'autant plus vraie que l'extension de chaque paquet est plus petite devant la distance entre paquets. $n$ et $p$ n'étant liés que par la relation $p n=N$, le système initial peut donc choisir parmi plusieurs configurations de paquets. Reste à savoir pourquoi il tend vers une configuration à un petit nombre de paquets.

Etudions donc les modes propres de vibration des tourbillons lorsqu'ils sont disposés aux sommets d'un polygone régulier de $N$ côtés. Soit $v_{l}(t)$ un petit déplacement du $l$-ième point par rapport à la position d'équilibre, défini par

$$
z_{l}=R \alpha_{l} \mathrm{e}^{i \omega t}\left(1+v_{l}(t)\right)
$$

où $\omega$ est la pulsation du mouvement circulaire uniforme (éq. (4)) et où les $\alpha$ sont les racines $N$-ièmes de l'unité

$$
\alpha_{l}=\exp (l 2 \pi i / N)
$$

Après linéarisation en $v$, les équations du mouvement s'écrivent :

$$
\begin{gathered}
\forall j=1, \ldots, N, \\
i \omega v_{j}+\frac{\mathrm{d} v_{j}}{\mathrm{~d} t}=-\frac{2 i \omega}{N-1} \sum_{l}, \frac{\bar{v}_{j}-\alpha_{j-l} \bar{v}_{l}}{\left(1-\alpha_{j-l}\right)^{2}} .
\end{gathered}
$$

L'invariance par rotation de $2 \pi / N$ suggère le changement de variables (transformation de Fourier) :

$$
\begin{array}{r}
\forall l \quad v_{l}(t)=\sum_{k=1}^{N}\left(A_{k} \exp \left(i k j \frac{2 \pi}{N}+i \Omega_{k} t\right)+\right. \\
\left.+B_{k} \exp \left(-i k j \frac{2 \pi}{N}-i \bar{\Omega}_{k} t\right)\right)
\end{array}
$$

qui scinde le système en $N$ couples d'équations

$$
\begin{gathered}
\forall k=1, \ldots, N, \\
\left(\omega+\Omega_{k}\right) A_{k}+\omega F(-k) \bar{B}_{k}=0 \\
\omega F(k) A_{k}+\left(\omega-\Omega_{k}\right) \bar{B}_{k}=0
\end{gathered}
$$

où la fonction $F$, définie par

$$
\forall k \in Z, \quad F(k)=\frac{2}{N-1} \sum_{m=1}^{N-1} \frac{1-\alpha_{m}^{k+1}}{\left(1-\alpha_{m}\right)^{2}}
$$

est réelle et a pour valeur

$$
F(k)=1-\frac{k(N-k)}{N-1} \text { pour } 0 \leqslant k \leqslant N .
$$

D’où les pulsations des $2 N$ modes propres :

$$
\begin{gathered}
\forall k=0, \ldots, N-1, \\
\left(\frac{\Omega_{k}}{\omega}\right)^{2}=\frac{k(N-k)}{N-1}\left(2-\frac{k(N-k)}{N-1}\right) .
\end{gathered}
$$

Si l'on exprime ce résultat en fonction du vecteur d'onde

$$
q=\frac{2 \pi k}{N a}
$$

on constate que le spectre ne dépend pas seulement du vecteur d'onde, comme c'est le cas par exemple pour les modes de vibration d'un réseau cristallin (phonons), mais encore, et d'une manière cruciale, du nombre de tourbillons :

$$
\forall k=0, \ldots, N-1,
$$

$$
\begin{aligned}
\left(\frac{\Omega_{k}}{\omega}\right)^{2}=\frac{N^{2}}{N-1} & \frac{q a}{2 \pi}\left(1-\frac{q a}{2 \pi}\right) \times \\
& \times\left(2-\frac{N^{2}}{N-1} \frac{q a}{2 \pi}\left(1-\frac{q a}{2 \pi}\right)\right) .
\end{aligned}
$$

\section{Tableau I}

Pulsations des modes, en unités $\omega$, pour $N$ inférieur ou égal à 7

$\begin{array}{cccccc}N & k & 0 & 1 & 2 & 3 \\ 3 & \frac{1}{2} & 0 & 1 & --- & --- \\ 4 & 0 & 1 & \frac{2 \sqrt{2}}{3} & --- \\ 5 & 0 & 1 & \frac{\sqrt{3}}{2} & --- \\ 6 & 0 & 1 & \frac{4}{5} & \frac{3}{5} \\ 7 & 0 & 1 & \frac{\sqrt{5}}{3} & 0\end{array}$


Il existe en particulier une valeur limite de $N$ au-delà de laquelle le spectre n'est plus entièrement réel; la fréquence limite a pour indice $k=[N / 2]$, ce qui fournit la valeur $N=7$.

4. Conclusion. - Ainsi, une telle nappe de tourbillons est instable pour de petites déformations. La vorticité, qui était initialement régulièrement répartie, tend à se concentrer en un nombre réduit d'endroits, nombre inférieur à 7 , ce qui constitue une violation de la symétrie de rotation. Expérimentalement on observe un nombre final de paquets égal à 5 ou à 6 mais pas à 2 ou à 3 par exemple. Il semble en effet que le système essaie de conserver la symétrie de rotation maximale.

Les études que nous poursuivons sur ce sujet s'attachent à la détermination de la structure interne d'un paquet, en suivant de près le processus d'enroulement de la nappe.

Remerciements. - Nous tenons à remercier ici le professeur de Gennes, qui a suggéré et guidé cette étude, et le professeur Guiraud qui a attiré notre attention sur certains points délicats.

\section{Bibliographie}

[1] Chorin, A. J. and Bernard, P. S., Discretization of a vortex sheet with an example of roll-up (1972), à paraître.

[2] MOORE, D. W., J. Fluid Mech. 63 (1973) 225.

[3] Nielsen, J. N. and SCHWind, R. G., Decay of a vortex pair behind an aircraft. Aircraft wake turbulence and its detection (1971) p. 413.

[4] Ralston, P. and WILF, H., ed. John Willey, Mathematical methods for digital computers, 1967, p. 95. 